\title{
REAL FIELDS, VALUATIONS, AND QUADRATIC FORMS
}

\author{
KARIM JOHANNES BECHER AND DAVID B. LEEP
}

\begin{abstract}
We study several field invariants arising in quadratic form theory. Some of the invariants considered are of particular interest in the study of real fields, including the length, the $u$-invariant, and the (reduced) stability index. In this context we give a systematic account of valuation theoretic arguments that lead to lower bounds for these invariants.

Keywords: quadratic form, real field, preordering, fan, Witt ring, powers of the fundamental ideal, real valuation, residue map, length, $u$-invariant, stability index, rational function field, power series field
\end{abstract}

Classification (MSC 2010): 11E04, 11E10, 11E81, 12D15

\section{INTRODUCTION}

This article studies the interplay between valuations and certain field invariants related to quadratic forms, with a special focus on phenomena that occur over (formally) real fields. The invariants considered include the $u$-invariant, the length, the stability index, and two further invariants related to the vanishing of certain ideals in the Witt ring of a field. One of our aims is to systematize the presentation of the usage of valuations to obtain lower bounds on these invariants.

We assume that the reader is familiar with the basic theory of quadratic forms over fields, for which we refer to [20]. By a 'form' or a 'quadratic form' we mean a regular quadratic form. We identify quadratic forms up to isometry and use the equality sign to indicate that two quadratic forms are isometric.

Let $K$ always be a field of characteristic different from 2. We denote by $K^{\times}$ the multiplicative group of $K$, by $K^{\times 2}$ the subgroup of nonzero squares in $K$, and by $\sum K^{2}$ the subgroup of nonzero sums of squares in $K$. By the Artin-Schreier Criterion (cf. [20, Chap. VIII, Sect. 1]) $K$ admits a field ordering if and only if $-1 \notin \sum K^{2}$; in this case the field $K$ is called (formally) real, otherwise nonreal. By $\mathbb{N}$ we denote the natural numbers including 0 . For $n \in \mathbb{N}$ and $a_{1}, \ldots, a_{n} \in K^{\times}$, we write $\left\langle\left\langle a_{1}, \ldots, a_{n}\right\rangle\right\rangle$ for the quadratic form $\left\langle 1,-a_{1}\right\rangle \otimes \cdots \otimes\left\langle 1,-a_{n}\right\rangle$ over $K$ and call this an $n$-fold Pfister form. Given a form $\varphi$ over $K$, we denote by $D_{K}(\varphi)$ the set of nonzero elements of $K$ represented by $\varphi$ and, for $n \in \mathbb{N}$, by $n \times \varphi$ the $n$-fold sum $\varphi \perp \ldots \perp \varphi$. If $\varphi=\left\langle a_{1}, \ldots, a_{n}\right\rangle$ for $a_{1}, \ldots, a_{n} \in K^{\times}$, we simply write $D_{K}\left\langle a_{1}, \ldots, a_{n}\right\rangle$ for $D_{K}(\varphi)$. If $D_{K}(\varphi) \subseteq \sum K^{2}$, then $\varphi$ is said to be totally positive, and if $D_{K}(\varphi)=\sum K^{2}$, then we say that $\varphi$ is positive-universal. We say

Date: June 13, 2011. 
that $\varphi$ is torsion if $n \times \varphi$ is hyperbolic for some integer $n \geq 1$. We denote the Witt equivalence class of the form $\varphi$ by $[\varphi]$.

We use the standard conventions for calculations involving $\infty$. For $N \subseteq \mathbb{N}$ we denote by $\inf N$ the infimum and by $\sup N$ the supremum of $N$, each taken in $\mathbb{N} \cup\{\infty\}$. In particular $\sup \emptyset=0, \inf \emptyset=\infty$, and $\sup \mathbb{N}=\infty$.

The length, the $u$-invariant and pythagoras number of $K$ are defined by

$$
\begin{aligned}
& \ell(K)=\inf \left\{n \in \mathbb{N} \mid \begin{array}{c}
\text { any totally positive form over } K \\
\text { of dimension } n \text { is positive-universal }
\end{array}\right\}, \\
& u(K)=\sup \{\operatorname{dim}(\varphi) \mid \varphi \text { is an anisotropic torsion form over } K\}, \\
& p(K)=\inf \left\{n \in \mathbb{N} \mid \text { any element of } \sum K^{2} \text { is a sum of } n \text { squares in } K\right\} .
\end{aligned}
$$

Whenever $K$ is nonreal, then every quadratic form over $K$ is totally positive and torsion so that $\ell(K)=u(K)$. For an overview on known results on these invariants, we refer to [25, Chap. 8] and [20, Chap. XI, Sect. 6] for the $u$-invariant, and further to [5] for the length.

The other invariants that we consider are intrinsically related to the powers of the fundamental ideal and related torsion ideals in the Witt ring. We denote by $W K$ the Witt ring of $K$, by $I K$ the fundamental ideal in $W K$, and by $I_{t} K$ the torsion part of $I K$. Let $n \geq 1$. We write $I^{n} K=(I K)^{n}$ and $I_{t}^{n} K=I^{n} K \cap I_{t} K$. If $K$ is nonreal, then $I_{t}^{n} K=\left(I_{t} K\right)^{n}=I^{n} K$. In general, we have that $\left(I_{t} K\right)^{n} \subseteq I_{t}^{n} K$. For a real field $K$, one may want to compare the ideals $\left(I_{t} K\right)^{n}$ and $I_{t}^{n} \bar{K}$, and in particular the conditions that $\left(I_{t} K\right)^{n}=0$ and that $I_{t}^{n} K=0$. To this aim it is very useful to know that both ideals are generated by $n$-fold Pfister forms of a particular shape. It is easy to see that the ideal $I^{n} K$ is generated as a group by the Witt equivalence classes of $n$-fold Pfister forms over $K$ and that $\left(I_{t} K\right)^{n}$ is generated as a group by the classes $\left[b\left\langle\left\langle a_{1}, \ldots, a_{n}\right\rangle\right\rangle\right]$ with $a_{1}, \ldots, a_{n} \in \sum K^{2}$ and $b \in K^{\times}$. Trivially, the ideal $I_{t} K \cdot I^{n-1} K$ is generated as a group by the classes of scaled $n$-fold Pfister forms $\left[b\left\langle\left\langle a_{1}, \ldots, a_{n}\right\rangle\right\rangle\right]$ with $a_{1} \in \sum K^{2}$ and $b, a_{2}, \ldots, a_{n} \in K^{\times}$. We thus have canonical generators for $I_{t}^{n} K$, in view of the following deep theorem.

\subsection{Theorem (Marshall-Orlov-Vishik-Voevodsky). For every $n \geq 1$ one has}

$$
I_{t}^{n} K=I_{t} K \cdot I^{n-1} K .
$$

In [21, Cor. 1] this was shown under the assumption that in the Milnor ring $k_{*} K$ the annihilator of a symbol is generated by elements of degree one, which was proved in $[24,(3.3)]$.

We now define two field invariants $\alpha$ and $\beta$ :

$$
\begin{aligned}
& \alpha(K)=\sup \left\{n \in \mathbb{N} \mid I_{t}^{n} K \neq 0\right\} \\
& \beta(K)=\sup \left\{n \in \mathbb{N} \mid\left(I_{t} K\right)^{n} \neq 0\right\}
\end{aligned}
$$

If $K$ is nonreal then $I_{t} K=I K$ and so $\alpha(K)=\beta(K)=\sup \left\{n \in \mathbb{N} \mid I^{n} K \neq 0\right\}$. The choice of the letters $\alpha$ and $\beta$ alludes to the Properties $\left(\mathrm{A}_{n}\right)$ and $\left(\mathrm{B}_{n}\right)$ introduced in [13]. With the terminology and notation of [13], in view of the above 
statements on ideal generators we have the following:

$$
\begin{aligned}
& \alpha(K)+1=\inf \left\{n \in \mathbb{N} \mid K \text { satisfies }\left(\mathrm{A}_{n}\right)\right\} \\
& \beta(K)+1=\inf \left\{n \in \mathbb{N} \mid K \text { satisfies }\left(\mathrm{B}_{n}\right)\right\}
\end{aligned}
$$

The equality relating $\alpha$ and $\left(A_{n}\right)$ relies on (1.1), whereas the equality relating $\beta$ and $\left(B_{n}\right)$ is far more elementary; see also [5, Sect. 2]. Furthermore, one has $\alpha(K)=\nu(K)-1$ for the $\nu$-invariant introduced in [13].

The following statement summarizes the relations that are known among the invariants introduced so far.

1.2. Proposition. We have $\alpha(K) \geq \beta(K) \geq 0$ and $\ell(K) \geq p(K) \geq 1$. If $p(K)>1$, then $u(K) \geq 2^{\alpha(K)}, \ell(K) \geq 2^{\beta(K)-1}+1$, and $p(K) \leq 2^{\beta(K)}$. Moreover, $p(K)=1$ if and only if $\ell(K)=1$ if and only if $\alpha(K)=0$ if and only if $\beta(K)=0$, and in this case $u(K)$ is either 0 or 1 depending on whether $K$ is real or nonreal.

Proof: The inequalities in the first sentence are trivial. The inequalities in the second sentence are immediate from [5, (2.7.a)], [5, (4.7.b)], and [5, (2.7.b)], respectively. The equivalences in the last sentence are clear from $[5,(4.1)]$, whereas the statement on the $u$-invariant is obtained from the proof of $[20$, Chap. XI, (6.26)].

Note that, if $p(K)=\ell(K)=2^{n-1}+1$ for some $n \geq 1$, then it follows from (1.2) that $\beta(K)=n$; examples with $n \leq 3$ are known, see [5, (7.3) and (7.4)].

We further consider the (reduced) stability index, introduced in [9]. This invariant is interesting for the study of real fields and has various characterizations. In Section 4 we revisit some crucial results from [9] on the stability index and explain how to derive these results from our study of real valuations in Section 3 and from Bröcker's 'Fan Trivialization Theorem' from [10], cited in (4.3) below. In contrast to [9] we do not use henselizations. In particular, in (4.5) we obtain a consequence of Bröcker's 'Global Stability Formula' [9, (3.19)] in a way that is appropriate for our applications, such as (6.8) below. In (4.1) we give the characterization of the stability index of $K$ as

$$
s t(K)=\inf \left\{r \in \mathbb{N} \mid I^{r+1} K=2 I^{r} K+I_{t}^{r+1} K\right\},
$$

which can be considered as a definition for the rest of the introduction.

The following theorem, pointed out in [18, Prop. 1] as a consequence of earlier results, relates the stability index to the invariant $\alpha$.

1.4. Theorem (Elman-Lam-Krüskemper). We have

$$
\alpha(K(\sqrt{-1}))=\max \{\alpha(K), \operatorname{st}(K)\} .
$$

For an exposition of the elementary but technical proof, see [11, Sect. 35.B] or [6].

In Section 5 we consider the situation of a valued field and use the residue homomorphism relating the Witt rings of the field and the corresponding residue field, and we provide lower bounds for the invariants under discussion. In the 
last two sections we apply these bounds to determine the invariants for special types of fields. In Section 6 we discuss the situation of a rational function field. With (6.8) below we obtain a proof of the following statement.

1.5. Theorem. Let $F=K(X)$. Then $\alpha(F)=\beta(F)=\alpha(F(\sqrt{-1}))$. Moreover, if $K$ is real, then $\alpha(F)=\sup \left\{n \in \mathbb{N} \mid\left(I_{t} F\right)^{n} \neq I_{t}^{n} F\right\}$.

In Section 7 we consider special extensions of a real closed field $R$. For a finitely generated field extension $F / R$ of transcendence degree $n$, we see in (7.2) that $\alpha(F)=\beta(F)=n$, and further $s t(F)=n$ when $F$ is real. Considering a field that is obtained from a real closed field by an iteration of power series field extensions and further one rational function field extension, we have a situation where all invariants under discussion here can be determined.

1.6. Theorem. Let $R$ be a real closed field, $m, n \in \mathbb{N}$ with $m \leq n$, and

$$
F=R\left(\left(t_{1}\right)\right) \ldots\left(\left(t_{m-1}\right)\right)\left(t_{m}\right)\left(\left(t_{m+1}\right)\right) \ldots\left(\left(t_{n}\right)\right) .
$$

Then $p(F)=2, \beta(F)=m, \ell(F)=2^{m}$, st $(F)=\alpha(F)=\alpha(F(\sqrt{-1}))=n$, and $u(F)=u(F(\sqrt{-1}))=2^{n}$.

The proof of (1.6) will be achieved in (7.3). From (1.6) we see in particular that there is no general upper bound on $\alpha(K)$ in terms of $\beta(K)$.

\section{FANS AND PYTHAGOREAN FIELDS}

A preordering of $K$ is a subset $T \subseteq K$ that $K^{2} \subseteq T, T+T \subseteq T, T \cdot T \subseteq T$, and $-1 \notin T$; if in addition $T \cup-T=K$, then $T$ is called an ordering of $K$. The set of all orderings of $K$ is denoted by $X_{K}$. For a preordering $T$ of $K$ we write $T^{\times}=T \backslash\{0\}$, which is a subgroup of $K^{\times}$, and $X_{T}=\left\{P \in X_{K} \mid T \subseteq P\right\}$. We use the notation $\pm S=S \cup-S$ for $S \subseteq K$.

Fans were introduced by Becker and Köpping in [8]; see also [19, Chap. 5]. They are characterized by the following proposition.

2.1. Proposition. Let $T$ be a preordering of $K$. The following are equivalent:

(i) For any subgroup $H$ of $K^{\times}$of index 2 containing $T^{\times}$and with $-1 \notin H$, the set $H \cup\{0\}$ is an ordering of $K$.

(ii) For any subgroup $H$ of $K^{\times}$containing $T^{\times}$and with $-1 \notin H$, the set $H \cup\{0\}$ is a preordering of $K$.

(iii) For any $a \in K^{\times} \backslash \pm T$ we have $T+a T=T \cup a T$.

Moreover, if $n \in \mathbb{N}$ is such that $\left[K^{\times}: T^{\times}\right]=2^{n}$, then $n \leq\left|X_{T}\right| \leq 2^{n-1}$, and the equality $\left|X_{T}\right|=2^{n-1}$ is equivalent to any of the conditions $(i)-($ iii $)$.

Proof: See [8, Satz 20] or [19, (5.1) and (5.5)].

A fan in $K$ is a preordering $T$ of $K$ for which the equivalent conditions $(i)-(i i i)$ in $(2.1)$ hold. For a fan $T$ we put $\operatorname{deg} T=\operatorname{dim}_{\mathbb{F}_{2}}\left(K^{\times} / \pm T^{\times}\right)=\operatorname{dim}_{\mathbb{F}_{2}}\left(K^{\times} / T^{\times}\right)-1$ and call this the degree of $T$. The orderings of $K$ are the fans of degree 0 . Any 
preordering $T$ of $K$ with $\left[K^{\times}: T^{\times}\right]=4$ is a fan of degree 1 . Fans of degree 0 or 1 are called trivial. Any preodering containing a fan is itself a fan. In particular, if there exists a fan of degree $n$ in $K$, then there exist fans of degree $m$ in $K$ for all natural numbers $m \leq n$.

The field $K$ is called pythagorean if $\sum K^{2}=K^{\times 2}$ (that is, $p(K)=1$ ), and euclidean if the set of squares $K^{2}$ is an ordering of $K$.

2.2. Proposition. Assume that $K$ is real. Then every real quadratic extension of $K$ is pythagorean if and only if $K^{2}$ is a fan in $K$.

Proof: See [19, (5.16)].

By a 2-extension we mean an algebraic extension $L / K$ such that, for any $\alpha \in L$ there exists $r \in \mathbb{N}$ and intermediate fields $K_{0}, \ldots, K_{r}$ such that $K_{0}=K, \alpha \in K_{r}$, and $K_{i} / K_{i-1}$ is a quadratic extension for $i=1, \ldots, r$. It follows from [19, (5.19)] that if $K^{2}$ is a fan in $K$ then $L^{2}$ is a fan in $L$ for any real 2-extension $L / K$. We will need a special case of this fact, contained in the following statement.

2.3. Proposition. If $K$ is nonreal with two square classes, then any finite 2extension of $K$ has two square classes. If $K$ is real pythagorean with four square classes, then any finite real 2-extension is pythagorean with four square classes and any finite nonreal 2-extension has two square classes.

Proof: For quadratic extensions the statement follows from the square class exact sequence in [20, Chap. VII, (3.8)]. This generalizes immediately to finite 2-extensions.

2.4. Proposition. Let $L / K$ be a finite extension and $P$ an ordering of $K$. The number of extensions of $P$ to an ordering of $L$ is bounded by $[L: K]$ and the two numbers are congruent modulo 2.

Proof: Let $R$ denote the real closure of $K$ with respect to $P$. Fix an irreducible polynomial $f \in K[X]$ such that $L$ is $K$-isomorphic to $K[X] /(f)$. The extensions of $P$ to an ordering of $L$ are given by the roots of $f$ in $R$ (cf. [26, (3.12)]). As $[L: K]$ is the number of roots of $f$ in the algebraically closed field $R(\sqrt{-1})$ and as the roots in $R(\sqrt{-1}) \backslash R$ are pairwise conjugate under the nontrivial automorphism of $R(\sqrt{-1}) / R$, the statement follows.

2.5. Corollary. Let $L / K$ be a finite extension of odd degree such that every real quadratic extension of $L$ is pythagorean. Then every ordering of $K$ extends uniquely to L.

Proof: Let $P$ be an ordering of $K$. Let $T$ be the preordering of $L$ generated by $P$. By (2.4) $X_{T}$ is finite of odd cardinality. By the hypothesis and $(2.2), L^{2}$ is a fan in $L$. Then also $T$ is a fan in $L$. Hence, $\left|X_{T}\right|$ is a power of 2 , by (2.1). We conclude that $\left|X_{T}\right|=1$. 
The field $K$ is said to be hereditarily pythagorean, respectively, hereditarily euclidean, if $K$ is real and if every finite real extension of $K$ is pythagorean, respectively, euclidean.

2.6. Theorem (Becker). Any uniquely ordered hereditarily pythagorean field is hereditarily euclidean.

Proof: Assume that $K$ is hereditarily pythagorean and uniquely ordered. Given a finite real extension $M / K$, let $N / K$ denote the normal closure of $M(\sqrt{-1}) / K$ and $L$ the fixed field of a 2-Sylow subgroup of the Galois group of $N / K$. Then $[L: K]$ is odd, so $L$ is real pythagorean and by (2.5) uniquely ordered. Hence, $L$ is euclidean. As $N / L$ is a nonreal 2-extension, it follows that $N=L(\sqrt{-1})$. Hence, $N$ is quadratically closed, and as $N / M(\sqrt{-1})$ is a finite extension, it follows by [20, Chap. VIII, (5.11)] that $M(\sqrt{-1})$ is quadratically closed, thus $M$ is euclidean by [20, Chap. VIII, (1.7)].

\section{Real valuations}

We collect some facts about ordered abelian groups and valuations. More details can be found in [15]. For any ring $R$ we denote by $R^{\times}$the multiplicative group of invertible elements in $R$. Given a valuation $v$ on $K$, we write $\mathcal{O}_{v}$ for the corresponding valuation ring, $\mathfrak{m}_{v}$ for its maximal ideal, $\kappa_{v}$ for the residue field $\mathcal{O}_{v} / \mathfrak{m}_{v}$, and $\Gamma_{v}$ for the value group, which is naturally isomorphic to $K^{\times} / \mathcal{O}_{v}^{\times}$.

We say that a valuation is real or nonreal, respectively, if the residue field has this property. We say that a valuation is dyadic if its residue field has characteristic two. We will only be interested in non-dyadic valuations. Real valuations are non-dyadic.

3.1. Proposition. A valuation $v$ on $K$ is real if and only if for any $r \geq 1$ and $x_{1}, \ldots, x_{r} \in K$ the equality $v\left(x_{1}^{2}+\cdots+x_{r}^{2}\right)=2 \min \left\{v\left(x_{1}\right), \ldots, v\left(x_{r}\right)\right\}$ holds.

Proof: This is easy to show.

Let $v$ be a valuation on $K$ and $\Delta$ a convex subgroup of the value group $\Gamma_{v}$. Composing $v$ with the quotient map $\Gamma_{v} \longrightarrow \Gamma_{v} / \Delta$, we obtain a valuation $v^{\prime}: K \longrightarrow\left(\Gamma_{v} / \Delta\right) \cup\{\infty\}$ with $\mathcal{O}_{v} \subseteq \mathcal{O}_{v^{\prime}}$, called a coarsening of $v$. Moreover, there is a unique valuation $\bar{v}: \kappa_{v^{\prime}} \longrightarrow \Delta \cup\{\infty\}$ such that $\bar{v}\left(x+\mathfrak{m}_{v^{\prime}}\right)=v(x)$ for $x \in \mathcal{O}_{v^{\prime}}^{\times}$. The corresponding valuation ring of $\kappa_{v^{\prime}}=\mathcal{O}_{v^{\prime}} / \mathfrak{m}_{v^{\prime}}$ is $\mathcal{O}_{\bar{v}}=\left(\mathcal{O}_{v}+\mathfrak{m}_{v^{\prime}}\right) / \mathfrak{m}_{v^{\prime}}$. Moreover, the places (residue maps) corresponding to $v, v^{\prime}$, and $\bar{v}$ are compatible in the sense that, identifying $\kappa_{v}=\mathcal{O}_{v} / \mathfrak{m}_{v}$ with $\kappa_{\bar{v}}=\left(\left(\mathcal{O}_{v}+\mathfrak{m}_{v^{\prime}}\right) / \mathfrak{m}_{v^{\prime}}\right) /\left(\mathfrak{m}_{v} / \mathfrak{m}_{v^{\prime}}\right)$, the residue map $\mathcal{O}_{v} \longrightarrow \kappa_{v}$ factors naturally over $\mathcal{O}_{\bar{v}}$. See also [15, p. 45].

Given a valuation $v$ on $K$, we denote by $\Sigma_{v}$ the subset of $\Gamma_{v}$ of elements of the form $\pm v(x)$ with $0 \neq x=x_{1}^{2}+\cdots+x_{r}^{2}$ for some $r \geq 1$ and $x_{1}, \ldots, x_{r} \in \mathcal{O}_{v}$ with $v\left(x_{1}\right)=0$. We set $\operatorname{rk}_{2}(v)=\operatorname{dim}_{\mathbb{F}_{2}}\left(\Gamma_{v} / 2 \Gamma_{v}\right)$ and call this the 2-rank of $v$. Hence, $\operatorname{rk}_{2}(v)=0$ if and only if $\Gamma_{v}=2 \Gamma_{v}$, that is, if and only if $\Gamma$ is 2-divisible. We set $\operatorname{nrk}_{2}(v)=\operatorname{dim}_{\mathbb{F}_{2}} v\left(\sum K^{2}\right) / 2 \Gamma_{v}$ and call this the nonreal 2-rank of $v$. 
3.2. Proposition. Let $v$ be a non-dyadic valuation on $K$. Then $\Sigma_{v}$ is a convex subgroup of $K$ and $v\left(\sum K^{2}\right)=\Sigma_{v}+2 \Gamma_{v}$. Let $v^{\prime}: K \longrightarrow\left(\Gamma_{v} / \Sigma_{v}\right) \cup\{\infty\}$ denote the induced coarsening of $v$, the residue field $\kappa_{v^{\prime}}$, and $\bar{v}: \kappa_{v^{\prime}}: \longrightarrow \Sigma_{v} \cup\{\infty\}$ the valuation induced by $v$ on $\kappa_{v^{\prime}}$. Then $\operatorname{nrk}_{2}(v)=\operatorname{rk}_{2}(\bar{v})$, and if $K$ is real, then $v^{\prime}$ is a real valuation. Moreover, $v$ is real if and only if $K$ is real and $\Sigma_{v}=0$.

Proof: Let $S$ be the set of all $x \in K^{\times}$of the form $x=x_{1}^{2}+\cdots+x_{r}^{2}$ with $r \geq 1$ and $x_{1}, \ldots, x_{r} \in \mathcal{O}_{v}$ such that $v\left(x_{1}\right)=0$. Note that $S$ is closed under multiplication and contains 1 . Hence, $\Sigma_{v}^{+}=v(S)$ is closed under addition and contains 0 . For $x \in S$ and $t \in K$ with $0<v(t)<v(x)$ we have $x^{\prime}=\left(x_{1}+t\right)^{2}+x_{2}^{2}+\cdots+x_{r}^{2} \in S$ and $v\left(x^{\prime}\right)=v(t)$. This shows that $\Sigma_{v}^{+}$is a convex subset of $\Gamma_{v}$. We conclude that $\Sigma_{v}=\Sigma_{v}^{+} \cup-\Sigma_{v}^{+}$is a convex subgroup of $\Gamma_{v}$. It is clear that $v\left(\sum K^{2}\right)=\Sigma_{v}+2 \Gamma_{v}$.

As $\Sigma_{v}$ is convex in $\Gamma_{v}$, we have $\Sigma_{v} \cap 2 \Gamma_{v}=2 \Sigma_{v}$, and therefore

$$
v\left(\sum K^{2}\right) / 2 \Gamma_{v}=\left(\Sigma_{v}+2 \Gamma_{v}\right) / 2 \Gamma_{v} \cong \Sigma_{v} / 2 \Sigma_{v} .
$$

As $\Sigma_{v}$ is the value group of $\bar{v}$, this shows that $\operatorname{nrk}_{2}(v)=\operatorname{rk}_{2}(\bar{v})$.

Obviously, if $v$ is real, then $K$ is real and $\Sigma_{v}=0$ by (3.1). Assume now that $K$ is real. Then for $r \geq 1$ and $x_{1}, \ldots, x_{r} \in K$ we have $v\left(x_{1}^{2}+\cdots+x_{r}^{2}\right) \equiv$ $2 \min \left\{v\left(x_{1}\right), \ldots, v\left(x_{r}\right)\right\} \bmod \Sigma_{v}$, thus $v^{\prime}\left(x_{1}^{2}+\cdots+x_{r}^{2}\right)=2 \min \left\{v^{\prime}\left(x_{1}\right), \ldots, v^{\prime}\left(x_{r}\right)\right\}$. Hence, $v^{\prime}$ is a real valuation by (3.1). In particular, if $\Sigma_{v}=0$, then $v$ is real.

3.3. Example. Let $v$ be a $\mathbb{Z}$-valuation on $K$. Then $v\left(\sum K^{2}\right)=2 \mathbb{Z}$ if $v$ is real, and $v\left(\sum K^{2}\right)=\mathbb{Z}$ otherwise.

In general we have $2 \Gamma_{v} \subseteq v\left(\sum K^{2}\right) \subseteq \Gamma_{v}$. If $K$ is real then $v\left(\sum K^{2}\right)=2 \Gamma_{v}$. If $v$ is nonreal, however, any of the inclusions may be or not be strict. For example, there is a valuation on $K=\mathbb{R}(X, Y)$ with residue field $\mathbb{C}$ and value group $\Gamma_{v}$ equal to $\mathbb{Z} \times \mathbb{Z}$ with the lexicographic order and where $2 \Gamma_{v} \subsetneq v\left(\sum K^{2}\right) \subsetneq \Gamma_{v}$. By contrast, in (5.3) below we give an example where $K=\mathbb{R}, \kappa_{v}$ is nonreal and $\Gamma_{v}$ is divisible, implying that $v\left(\sum K^{2}\right)=\Gamma_{v}=2 \Gamma_{v}$.

The following is a slight strengthening of $[9,(3.2)]$.

3.4. Lemma. Let $\Gamma$ be a torsion free abelian group and $\Delta \subseteq \Gamma$ a subgroup such that $\Gamma / \Delta$ is a torsion group. Then for $n \geq 1$, we have $|\Gamma / n \Gamma| \leq|\Delta / n \Delta|$, with equality holding if $\Gamma / \Delta$ is finite.

Proof: $\quad$ Let $n \geq 1$. Note that $|\Gamma / n \Gamma| \cdot|n \Gamma / n \Delta|=|\Gamma / n \Delta|=|\Gamma / \Delta| \cdot|\Delta / n \Delta|$. Assume first that $\Gamma / \Delta$ is finite. Then the natural surjection $\Gamma / \Delta \longrightarrow n \Gamma / n \Delta$ induced by multiplication by $n$ is an isomorphism as $\Gamma$ is torsion free, hence $|\Gamma / \Delta|=|n \Gamma / n \Delta|$ and thus $|\Gamma / n \Gamma|=|\Delta / n \Delta|$.

In the general case, for any $r \in \mathbb{N}$ with $r \leq|\Gamma / n \Gamma|$, the subgroup $\Gamma^{\prime}$ of $\Gamma$ generated by $\Delta$ and representatives of $r$ distinct classes in $\Gamma / n \Gamma$ is such that $\Gamma^{\prime} / \Delta$ is finite, which implies that $r \leq\left|\Gamma^{\prime} / n \Gamma^{\prime}\right|=|\Delta / n \Delta|$ by the previous case. Therefore we have $|\Gamma / n \Gamma| \leq|\Delta / n \Delta|$ in general. 
To see that the inequality in (3.4) may be strict when $\Gamma / \Delta$ is not finite, one may take $\Gamma=\mathbb{Q}$ and $\Delta=\mathbb{Z}$.

For a field extension $L / K$ we denote by $\operatorname{td}(L / K)$ the transcendence degree.

3.5. Proposition. Let $L / K$ be field extension and $v$ a valuation of $L$. Then

$$
\operatorname{rk}_{2}(v) \leq \operatorname{rk}_{2}\left(\left.v\right|_{K}\right)+\operatorname{td}(L / K) .
$$

Proof: By (3.4) we have $\operatorname{rk}_{2}(v) \leq \operatorname{rk}_{2}\left(\left.v\right|_{K}\right)$ whenever $v\left(L^{\times}\right) / v\left(K^{\times}\right)$is torsion. Assume that $v\left(L^{\times}\right) / v\left(K^{\times}\right)$is not torsion. Then there exists $x \in L^{\times}$such that $n v(x) \notin v\left(K^{\times}\right)$for any $n \in \mathbb{N}$. Then $x$ is transcendental over $K$ by [15, (3.2.4)]. For $K^{\prime}=K(x)$ we thus have $\operatorname{td}\left(L / K^{\prime}\right)=\operatorname{td}(L / K)-1$. Furthermore $v\left(K^{\prime \times}\right)$ is generated by $v\left(K^{\times}\right)$and $v(x)$, so that $\operatorname{rk}_{2}\left(\left.v\right|_{K^{\prime}}\right)=\operatorname{rk}_{2}(v)+1$.

If $\operatorname{td}(L / K)<\infty$, we may thus conclude the claimed inequality by induction on $\operatorname{td}(L / K)$, whereas it trivially holds if $\operatorname{td}(L / K)=\infty$.

\section{Stability index, VAluations, And FAns}

We define the stability index of $K$ as

$$
\operatorname{st}(K)=\sup \{\operatorname{deg} T \mid T \text { fan in } K\} .
$$

Note that $s t(K)=0$ if and only if $K$ is either nonreal or uniquely ordered.

It follows from $[10,(2.11)]$ that this definition of the stability index is equivalent to the original definition in [9] and therefore to the definition given in (1.3).

4.1. Theorem (Bröcker). We have st $(K)=\inf \left\{r \in \mathbb{N} \mid I^{r+1} K=2 I^{r} K+I_{t}^{r+1} K\right\}$.

Proof: The original proof in $[10,(2.11)]$ involves valuations. A proof independent of valuation theory is contained in [22, Sect. 6].

In the remainder of this section we discuss the characterization of the stability index in terms of valuations, relying on Bröcker's 'Fan Trivialization Theorem' from [10] cited in (4.3) below, and we retrieve some crucial results from [9].

The following is implicit in [9, (3.3) and (3.18)].

4.2. Proposition. Let $v$ be a real valuation on $K$. Then $s t(K) \geq \operatorname{rk}_{2}(v)+s t\left(\kappa_{v}\right)$.

Proof: Let $T$ be a preordering of $\kappa_{v}$. Let $\widetilde{T}=\left\{t \in \mathcal{O}_{v}^{\times} \mid t+\mathfrak{m}_{v} \in T^{\times}\right\}$and $S=\left(K^{\times 2} \cdot \widetilde{T}\right) \cup\{0\}$. Then $S$ is a preordering of $K$ and $S^{\times} \cap \mathcal{O}_{v}^{\times}=\widetilde{T}$. It follows that $\left[K^{\times 2} \mathcal{O}_{v}^{\times}: S^{\times}\right]=\left[S^{\times} \mathcal{O}_{v}^{\times}: S^{\times}\right]=\left[\mathcal{O}_{v}^{\times}: \widetilde{T}\right]=\left[\kappa_{v}^{\times}: T^{\times}\right]$and therefore

$$
\left[K^{\times}: S^{\times}\right]=\left[K^{\times}: K^{\times 2} \mathcal{O}_{v}^{\times}\right] \cdot\left[K^{\times 2} \mathcal{O}_{v}^{\times}: S^{\times}\right]=\left[\Gamma_{v}: 2 \Gamma_{v}\right] \cdot\left[\kappa_{v}^{\times}: T^{\times}\right] .
$$

Furthermore, if $T$ is a fan, then so is $S$. From this, the claim follows.

A preordering $T$ of $K$ and a valuation $v$ on $K$ are said to be compatible with each other if $1+\mathfrak{m}_{v} \subseteq T$; this is equivalent to saying that $\left\{\bar{x} \mid x \in \mathcal{O}_{v}^{\times} \cap T^{\times}\right\} \cup\{0\}$ is a preordering of $\kappa_{v}$, then denoted by $\bar{T}_{v}$ and called the preordering induced by $T$; note that in this case we have $\left[K^{\times}: T^{\times}\right]=\left[\kappa_{v}^{\times}: \bar{T}^{\times}\right] \cdot\left[\Gamma_{v}: v\left(T^{\times}\right)\right]$. Note that 
our notion of 'compatibility' corresponds to 'full compatibility' in the terminology of $[19$, Sect. 3].

If a fan $T$ in $K$ is compatible with a valuation $v$ on $K$, then $\bar{T}_{v}$ is a fan in $\kappa_{v}$, and as $\left[K^{\times}: T^{\times}\right]=\left[\kappa_{v}^{\times}: \bar{T}^{\times}\right] \cdot\left[\Gamma_{v}: v\left(T^{\times}\right)\right]$, it follows that $\operatorname{deg} T-\operatorname{deg} \bar{T} \leq \operatorname{rk}_{2}(v)$.

4.3. Theorem (Bröcker). Every fan $T$ in $K$ is compatible with some real valuation $v$ on $K$ such that the induced fan $\bar{T}$ in $\kappa_{v}$ is trivial.

Proof: See $[10,(2.7)]$ for the original proof. A constructive proof is given in $[16$, Sect. 1]. See [23, Sect. 3.6] for another treatment.

4.4. Corollary. Assume that $K$ is real and st $(K)<\infty$. Then there exists a real valuation $v$ on $K$ such that $s t\left(\kappa_{v}\right)=\operatorname{st}(K)-\operatorname{rk}_{2}(v) \leq 1$.

Proof: $\quad$ Let $T$ be a fan of $K$ with $\operatorname{deg} T=\operatorname{st}(K)$. By (4.3) $T$ is compatible with a valuation $v$ on $K$ such that the induced fan $\bar{T}$ in $\kappa_{v}$ is trivial. With (4.2) we obtain that $\operatorname{deg} T=\operatorname{st}(K) \geq \operatorname{st}\left(\kappa_{v}\right)+\operatorname{rk}_{2}(v) \geq \operatorname{deg} \bar{T}+\operatorname{rk}_{2}(v) \geq \operatorname{deg} T$ and therefore $s t\left(\kappa_{v}\right)=\operatorname{st}(K)-\operatorname{rk}_{2}(v)=\operatorname{deg} T-\operatorname{rk}_{2}(v)=\operatorname{deg} \bar{T} \leq 1$.

We come to a characterization of the stability index in terms of valuations. It can also be derived from Bröcker's 'Global Stability Formula' [9, (3.19)], which characterizes the stability index of a field in terms of henselizations.

4.5. Theorem (Bröcker). Assume that $K$ is real. Let $N$ be the set of natural numbers $n$ with the property that any real valuation $v$ on $K$ satisfies $\operatorname{rk}_{2}(v) \leq n$ and, furthermore, $\operatorname{rk}_{2}(v)=n$ only if $\kappa_{v}$ is uniquely ordered. Then st $(K)=\inf N$.

Proof: For a real valuation $v$ on $K$ we have $\operatorname{rk}_{2}(v) \leq s t(K)-\operatorname{st}\left(\kappa_{v}\right)$ by (4.2), so that either $\operatorname{rk}_{2}(v)=s t(K)$ and $\kappa_{v}$ is uniquely ordered, or $\operatorname{rk}_{2}(v) \leq s t(K)-1$. Hence, either $\operatorname{st}(K)=\infty$ or $\operatorname{st}(K) \in N$, showing that inf $N \leq s t(K)$ in any case. Suppose now that inf $N<\operatorname{st}(K)$. Then there exists $n \in N$ and a fan $T$ in $K$ with $\operatorname{deg}(T)=n+1$. By (4.3) $T$ is compatible with a real valuation $v$ on $K$ such that the induced fan $\bar{T}$ in $\kappa_{v}$ is trivial. Then $\operatorname{rk}_{2}(v) \geq \operatorname{deg} T-\operatorname{deg} \bar{T} \geq n$. Moreover, if $\kappa_{v}$ is uniquely ordered, then $\operatorname{deg} \bar{T}=0$, so that $\operatorname{rk}_{2}(v) \geq \operatorname{deg} T=n+1$. This contradicts the fact that $n \in N$.

As an illustration of the applicability of (4.5) we recover $[9,(4.8)]$ :

4.6. Corollary (Bröcker). For any field extension $L / K$ one has

$$
\operatorname{st}(L) \leq \operatorname{st}(K)+\operatorname{td}(L / K)+1 .
$$

Proof: By (3.5) we have $\operatorname{rk}_{2}(v) \leq \operatorname{rk}_{2}\left(\left.v\right|_{K}\right)+\operatorname{td}(L / K)$ holds for any valuation $v$ on $L$. In view of this, the statement follows from (4.5).

Assuming that $\operatorname{st}(K)=n$, it follows from (4.5) that $K$ carries a real valuation $v$ with $\operatorname{rk}_{2}(v) \in\{n-1, n\}$. However, for $r \in\{n-1, n\}$ we can not say that a real valuation $v$ on $K$ with $\operatorname{rk}_{2}(v)=r$ will exist, as the following results show. 
4.7. Example. Let $r \in \mathbb{N}$ and $K=\mathbb{Q}(\sqrt{2})\left(\left(t_{1}\right)\right) \ldots\left(\left(t_{r}\right)\right)$. Then we have $\operatorname{rk}_{2}(v) \leq r$ for any real valuation $v$ on $K$. However, $\sum K^{2} \cup\{0\}$ is a fan of degree $r+1$ in $K$, in particular $s t(K)=r+1$. See also (7.1) for another argument.

Given an ordered group $G$ and a field $k$ we denote by $k((G))$ the field of formal power series as defined in $[15,(3.5 .6)]$, whose elements are formal sums $x=$ $\sum_{\gamma \in G} x_{\gamma} t^{\gamma}$ with $\left(x_{\gamma}\right) \in k^{G}$ whose support $\left\{\gamma \in G \mid x_{\gamma} \neq 0\right\}$ is well-ordered, with field operations defined in the obvious way. The elements of $k((G))$ whose support is contained in the nonnegative part of $G$ form a valuation ring denoted $k \llbracket G \rrbracket$, which is henselian.

We are grateful to A. Prestel for providing us with the following observation.

4.8. Proposition. Let $G$ be an archimedian ordered group and $K=\mathbb{R}((G))$. Then $\mathbb{R} \llbracket G \rrbracket$ is the only nontrivial valuation ring of $K$ with a real residue field. In particular, $s t(K)=\operatorname{dim}_{\mathbb{F}_{2}} G / 2 G$.

Proof: As $G$ is archimedian ordered, it contains no nontrivial convex subgroup, and therefore the valuation ring $\mathbb{R} \llbracket G \rrbracket$ of $K$ is maximal by $[15,(2.3 .2)]$. Let $w$ be a nontrivial real valuation on $K$. By $[15,(2.2 .5)]$ the valuation ring $\mathcal{O}_{w}$ is convex with respect to some ordering $P$ of $K$. Since $\mathbb{R} \llbracket G \rrbracket$ is a henselian valuation ring, it is also convex with respect to $P$, by $[15,(4.3 .6)]$. Hence, the valuation ring of $K$ obtained as the convex closure of $\mathbb{Z}$ with respect to $P$ is contained in $\mathcal{O}_{w}$ and in $\mathbb{R} \llbracket G \rrbracket$. It follows by $[15$, Sect. 2.3$]$ that the two valuation rings $\mathcal{O}_{w}$ and $\mathbb{R} \llbracket G \rrbracket$ of $K$ are dependent and therefore comparable. Since $\mathbb{R} \llbracket G \rrbracket$ is a maximal proper subring of $K$ we thus have $\mathcal{O}_{w} \subseteq \mathbb{R} \llbracket G \rrbracket$. Hence, $w$ induces a valuation $\widetilde{w}$ on the residue field $\mathbb{R}$ of $\mathbb{R} \llbracket G \rrbracket$. As $\widetilde{w}$ has the same residue field as $w$, it is also a real valuation. However, $\mathbb{R}$ carries no nontrivial real valuation by $[15,(2.2 .6)]$. Hence $\widetilde{w}$ is trivial and thus $\mathcal{O}_{w}=\mathbb{R} \llbracket G \rrbracket$.

This shows that $\mathbb{R} \llbracket G \rrbracket$ is the unique nontrivial valuation ring of $K$ with a real residue field. Let $v$ be the valuation corresponding to $\mathbb{R} \llbracket G \rrbracket$ with value group $G$. As its residue field $\mathbb{R}$ is uniquely ordered, we obtain that $\operatorname{st}(K)=\operatorname{rk}_{2}(v)=$ $\operatorname{dim}_{\mathbb{F}_{2}} G / 2 G$ by (4.5).

By an appropriate choice of $G$ in (4.8), we obtain for any $n \in \mathbb{N}$ a field $K$ carrying a unique nontrivial real valuation $v$ and such that $s t(K)=\operatorname{rk}_{2}(v)=n$.

\section{RESIDUE HOMOMORPHISMS}

Let $v$ in the sequel denote a non-dyadic valuation on $K$. By [17, (3.1)], there is a unique group homomorphism

$$
\partial_{v}: W K \longrightarrow W \kappa_{v}
$$

such that $\partial_{v}\left\langle u t^{2}\right\rangle=\langle\bar{u}\rangle$ for any $t, u \in K^{\times}$with $v(u)=0$, and $\partial_{v}\langle y\rangle=0$ for any $y \in K^{\times}$with $v(y) \notin 2 \Gamma_{v}$. Given a form $\varphi$ over $K$, we write $\partial_{v}(\varphi)$ instead of $\partial_{v}([\varphi])$. We say that $\varphi$ is $v$-balanced if $\varphi=\left\langle x_{1}, \ldots, x_{m}\right\rangle$ with $m \in \mathbb{N}$ and 
$x_{1}, \ldots, x_{m} \in K^{\times}$such that $v\left(x_{i}\right) \not \equiv v\left(x_{j}\right) \bmod 2 \Gamma_{v}$ for $1 \leq i<j \leq m$; this does not depend on the diagonalization, and we have $m=\operatorname{dim}(\varphi) \leq 2^{\mathrm{rk}_{2}(v)}$.

In the sequel, let $r, n \in \mathbb{N}$. There exists a $v$-balanced $n$-fold Pfister form over $K$ if and only if $n \leq \operatorname{rk}_{2}(v)$. Moreover, there exists a $v$-balanced totally positive $n$-fold Pfister form over $K$ if and only if $n \leq \operatorname{nrk}_{2}(v)$. Furthermore, any $n$-fold Pfister form over $K$ is of the shape $\left\langle\left\langle a_{1}, \ldots, a_{n}\right\rangle\right\rangle$ with $a_{1}, \ldots, a_{n} \in K^{\times}$such that $a_{i} \in \mathcal{O}_{v}^{\times}$for $\operatorname{rk}_{2}(v)<i \leq n$.

5.1. Lemma. Let $a_{1}, \ldots, a_{n} \in \mathcal{O}_{v}^{\times}$be such that $\left\langle\overline{a_{1}}, \ldots, \overline{a_{n}}\right\rangle$ over $\kappa_{v}$ is anisotropic. Then $\varphi \otimes\left\langle a_{1}, \ldots, a_{n}\right\rangle$ is anisotropic for any $v$-balanced form $\varphi$ over $K$.

Proof: Let $\psi=\left\langle a_{1}, \ldots, a_{n}\right\rangle$. By the hypothesis, $\psi$ is anisotropic over $K$ and $v\left(D_{K}(\psi)\right)=2 \Gamma_{v}$. Write $\varphi=\left\langle b_{1}, \ldots, b_{r}\right\rangle$ with $r \in \mathbb{N}$ and $b_{1}, \ldots, b_{r} \in K^{\times}$. As $v\left(D_{K}\left(b_{i} \psi\right)\right)=v\left(b_{i}\right)+2 \Gamma_{v}$ for $i=1, \ldots, r$ and $v\left(b_{i}\right) \not \equiv v\left(b_{j}\right) \bmod 2 \Gamma_{v}$ for $1 \leq i<j \leq r$, it follows that $\varphi \otimes \psi=b_{1} \psi \perp \ldots \perp b_{r} \psi$ is anisotropic over $K$.

For $x \in \mathbb{R}$ we use the notation $\lfloor x\rfloor=\max \{z \in \mathbb{Z} \mid z \leq x\}$.

5.2. Theorem. We have $u(K) \geq 2^{\mathrm{rk}_{2}(v)+1} \cdot\left\lfloor\frac{u\left(\kappa_{v}\right)}{2}\right\rfloor$ and $\ell(K) \geq 2^{\mathrm{nrk}_{2}(v)} \cdot \ell\left(\kappa_{v}\right)$. Moreover, $u(K) \geq 2^{\mathrm{rk}_{2}(v)} \cdot u\left(\kappa_{v}\right)$ except possibly when $K$ is real, $u\left(\kappa_{v}\right)$ is odd, and $\operatorname{nrk}_{2}(v)=0$.

Proof: Let $\kappa=\kappa_{v}$ and $\mathcal{O}=\mathcal{O}_{v}$. Let $\rho$ be an anisotropic torsion form of even dimension over $\kappa$. Then $\rho$ is Witt equivalent to a sum of torsion binary forms over $\kappa$, which can be written as $\bar{b}_{1}\left\langle 1,-\bar{s}_{1}\right\rangle \perp \cdots \perp \bar{b}_{n}\left\langle 1,-\bar{s}_{n}\right\rangle$ with $n \geq 0$, $b_{1}, \ldots, b_{n} \in \mathcal{O}^{\times}$and $s_{1}, \ldots, s_{n} \in \mathcal{O}^{\times} \cap \sum K^{2}$. Let $\varphi$ be the anisotropic part of the form $b_{1}\left\langle 1,-s_{1}\right\rangle \perp \cdots \perp b_{n}\left\langle 1,-s_{n}\right\rangle$ over $K$. Note that $\varphi$ is a torsion form and that $\operatorname{dim}(\varphi) \geq \operatorname{dim}(\rho)$, as $\rho$ is the anisotropic representative of the class $\partial_{v, 1}(\varphi)$ in $W \kappa$. For any $r \leq \operatorname{rk}_{2}(v)$ there is a $v$-balanced $r$-fold Pfister form $\pi$ over $K$, and then $\psi=\pi \otimes \varphi$ is an anisotropic torsion form over $K$ with $\operatorname{dim}(\psi)=2^{r} \operatorname{dim}(\varphi) \geq 2^{r} \operatorname{dim}(\rho)$. This shows that $u(K) \geq 2^{\mathrm{rk}_{2}(v)} \cdot 2 \cdot\left\lfloor\frac{u(\kappa)}{2}\right\rfloor$.

If $u(\kappa)$ is even, then $2 \cdot\left\lfloor\frac{u(\kappa)}{2}\right\rfloor=u(\kappa)$. Assume now that either $K$ is nonreal or $\operatorname{nrk}_{2}(v)>0$. We choose a $v$-balanced torsion $r$-fold Pfister form $\pi$ as follows. If $K$ is nonreal, any $v$-balanced $r$-fold Pfister form over $K$ is torsion. If $\operatorname{nrk}_{2}(v)>0$, then $2 \Gamma \subsetneq v\left(\sum K^{2}\right)$, so we choose $a_{1} \in \sum K^{2}$ and $a_{2}, \ldots, a_{r} \in K^{\times}$such that the classes of $v\left(a_{1}\right), \ldots, v\left(a_{r}\right)$ in $\Gamma / 2 \Gamma$ are $\mathbb{F}_{2}$-independent, and put $\pi=\left\langle\left\langle a_{1}, \ldots, a_{r}\right\rangle\right\rangle$. Now, for an arbitrary anisotropic form $\varphi$ over $\kappa$, we choose $b_{1}, \ldots, b_{n} \in \mathcal{O}^{\times}$such that $\varphi=\left\langle\bar{b}_{1}, \ldots, \bar{b}_{n}\right\rangle$, and then $\pi \otimes\left\langle b_{1}, \ldots, b_{n}\right\rangle$ is an anisotropic torsion form over $K$. Therefore, the assumption implies that $u(K) \geq 2^{\mathrm{rk}_{2}(v)} \cdot u(\kappa)$.

Consider $n, r \in \mathbb{N}$ with $n \leq \ell(\kappa)$ and $r \leq \operatorname{nrk}_{2}(v)$. There is a $v$-balanced totally positive $r$-fold Pfister form $\pi=\langle 1\rangle \perp \overline{\pi^{\prime}}$ over $K$ and $b_{1}, \ldots, b_{n} \in \sum K^{2} \cap \mathcal{O}^{\times}$ such that $\left\langle\bar{b}_{1}, \ldots, \bar{b}_{n-1},-\bar{b}_{n}\right\rangle$ over $\kappa$ is anisotropic. If $\kappa$ is real, then the form $\pi^{\prime} \otimes\left\langle b_{1}, \ldots, b_{n}\right\rangle \perp\left\langle b_{1}, \ldots, b_{n-1}\right\rangle$ over $K$ is totally positive and does not represent $b_{n}$. If $\kappa$ is nonreal, there exists $b \in \sum K^{2} \cap \mathcal{O}^{\times}$such that $\bar{b}=-\bar{b}_{n}$, and then the form $\pi^{\prime} \otimes\left\langle b_{1}, \ldots, b_{n-1}, b\right\rangle \perp\left\langle b_{1}, \ldots, b_{n-1}\right\rangle$ over $K$ is totally positive and does not 
represent $b_{n}$. In either case we conclude that $\ell(K) \geq 2^{r} n$. As this holds for all $n \leq \ell(\kappa)$ and $r \leq \operatorname{nrk}_{2}(v)$, we have $\ell(K) \geq 2^{\text {nrk }_{2}(v)} \cdot \ell(\kappa)$.

The 'integral part function' cannot be omitted in the lower bound for the $u$ invariant in (5.2), as the next example shows.

5.3. Example. Let $K$ be a real closed field and $v$ be any extension of a $p$-adic valuation of $\mathbb{Q}$ to $K$ for an odd prime number $p$. Then the residue field $\kappa_{v}$ is algebraically closed, so $u\left(\kappa_{v}\right)=1$. On the other hand, $K$ is real pythagorean, so $u(K)=0$. The value group $\Gamma_{v}$ is divisible, so $\operatorname{rk}_{2}(v)=0$. Hence, we have $u(K)=2^{\mathrm{rk}_{2}(v)} \cdot 2 \cdot\left\lfloor\frac{u\left(\kappa_{v}\right)}{2}\right\rfloor<2^{\mathrm{rk}_{2}(v)} \cdot u\left(\kappa_{v}\right)$ in this situation.

5.4. Proposition. Assume that $v$ is real. Then $\partial_{v}\left(I_{t} K\right)^{n}=\left(I_{t} \kappa_{v}\right)^{n}$.

Proof: As $\kappa_{v}$ is real, we have $v\left(\sum K^{2}\right)=v\left(K^{\times 2}\right)$. Hence $\left(I_{t} K\right)^{n}$ is generated as a group by the elements $\left[c\left\langle\left\langle a_{1}, \ldots, a_{n}\right\rangle\right\rangle\right]$ with $c \in K^{\times}$and $a_{1}, \ldots, a_{n} \in \mathcal{O}_{v}^{\times} \cap \sum K^{2}$. This yields the statement.

5.5. Proposition. Let $n, r \in \mathbb{N}$ with $n \geq 1$. Let $\pi$ be a $v$-balanced $r$-fold Pfister form over $K$.

(a) We have $I^{n} \kappa_{v}=\partial_{v}\left([\pi] \cdot I^{n} K\right) \subseteq \partial_{v}\left(I^{n+r} K\right)$.

(b) We have $I_{t}^{n} \kappa_{v}=\partial_{v}\left([\pi] \cdot I_{t}^{n} K\right) \subseteq \partial_{v}\left(I_{t}^{n+r} K\right)$.

(c) If $\pi$ is totally positive, then $\left(I_{t} \kappa_{v}\right)^{n}=\partial_{v}\left([\pi] \cdot\left(I_{t} K\right)^{n}\right) \subseteq \partial_{v}\left(\left(I_{t} K\right)^{n+r}\right)$.

Proof: The ideal $I^{n} \kappa_{v}$ is additively generated by the elements $\left[\bar{a}_{0}\left\langle\left\langle\bar{a}_{1}, \ldots, \bar{a}_{n}\right\rangle\right\rangle\right]$ where $a_{0}, \ldots, a_{n} \in \mathcal{O}_{v}^{\times}$. Similarly, $\left(I_{t} \kappa_{v}\right)^{n}$ is additively generated by the elements $\left[\bar{a}_{0}\left\langle\left\langle\bar{a}_{1}, \ldots, \bar{a}_{n}\right\rangle\right\rangle\right]$ where $a_{0} \in \mathcal{O}_{v}^{\times}$and $a_{1}, \ldots, a_{n} \in \mathcal{O}_{v}^{\times} \cap \sum K^{2}$. Finally by (1.1), $I_{t}^{n} \kappa_{v}$ is additively generated by the elements $\left[\bar{a}_{0}\left\langle\left\langle\bar{a}_{1}, \ldots, \bar{a}_{n}\right\rangle\right\rangle\right]$ with $a_{1} \in \sum K^{2} \cap \mathcal{O}_{v}^{\times}$ and $a_{0}, a_{2}, \ldots, a_{n} \in \mathcal{O}_{v}^{\times}$. Using these generators, the statements easily follow.

5.6. Corollary. We have $\beta(K) \geq \operatorname{nrk}_{2}(v)+\beta\left(\kappa_{v}\right)$.

Proof: For any $r \leq \operatorname{nrk}_{2}(v)$ there exists a totally positive $v$-balanced $r$-fold Pfister form $\pi$ over $K$. Hence, the statement follows from part $(c)$ of (5.5).

5.7. Corollary. Let $r=\operatorname{rk}_{2}(v)<\infty$. Then $\partial_{v}\left(I^{n+r} K\right)=I^{n} \kappa_{v}$ for $n \geq 0$ and $\partial_{v}\left(I_{t}^{n+r} K\right)=I_{t}^{n} \kappa_{v}$ for $n \geq 1$.

Proof: As $I^{n+r} K$ is additively generated by the elements $\left[a_{0}\left\langle\left\langle a_{1}, \ldots, a_{n+r}\right\rangle\right\rangle\right]$ with $a_{0}, \ldots, a_{r} \in K^{\times}$and $a_{r+1}, \ldots, a_{n+r} \in \mathcal{O}_{v}^{\times}$, it is clear that $\partial_{v}\left(I^{n+r} K\right) \subseteq I^{n} \kappa_{v}$. Since $\partial_{v}$ is a homomorphism it follows that $\partial_{v}\left(I_{t}^{n+r} K\right) \subseteq I_{t}^{n} \kappa_{v}$. The converse inclusions follow from parts $(a)$ and $(b)$ of (5.5) applied to an arbitrary $v$-balanced $r$-fold Pfister form $\pi$ over $K$.

5.8. Proposition. Assume that $v$ is real and $\kappa_{v}$ is not pythagorean. Then we have $\alpha(K) \geq \operatorname{rk}_{2}(v)+\alpha\left(\kappa_{v}\right)$. Moreover, if $\operatorname{rk}_{2}(v)>0$, then $\left(I_{t} K\right)^{m} \subsetneq I_{t}^{m} K$ for any $m \in \mathbb{N}$ with $2 \leq m \leq \alpha\left(\kappa_{v}\right)+\operatorname{rk}_{2}(v)$. 
Proof: By the hypotheses we have $\alpha\left(\kappa_{v}\right)>0$. Consider $r \in \mathbb{N}$ with $r \leq \operatorname{rk}_{2}(v)$ and choose a $v$-balanced $r$-fold Pfister form $\pi$ over $K$. Applying part (b) of (5.5), it follows that $I_{t}^{n} \kappa_{v} \subseteq \partial_{v}\left(I_{t}^{n+r} K\right)$ for any $n \geq 1$. Therefore $\alpha(K) \geq \alpha\left(\kappa_{v}\right)+r$. This shows the first part of the statement.

To prove the second part, suppose $m=n+r$ where $1 \leq n \leq \alpha\left(\kappa_{v}\right)$ and $1 \leq r \leq \operatorname{rk}_{2}(v)$. Then $I_{t}^{m} \kappa_{v} \subsetneq I_{t}^{n} \kappa_{v}$, by (1.1) and the Arason-Pfister Hauptsatz [20, Chap. X, (5.1)]. Using (5.4) we obtain that

$$
\partial_{v}\left(I_{t} K\right)^{m}=\left(I_{t} \kappa_{v}\right)^{m} \subseteq I_{t}^{m} \kappa_{v} \subsetneq I_{t}^{n} \kappa_{v} \subseteq \partial_{v}\left(I_{t}^{m} K\right),
$$

showing that $\left(I_{t} K\right)^{m} \subsetneq I_{t}^{m} K$.

Note that (5.8) and the second part of (5.7) depend on the deep result (1.1).

\section{RATIONAL FUNCTION FIELDS}

We study the special case of a rational function field $K(X)$.

6.1. Proposition. For $F=K(X)$ we have the following inequalities:

$$
\begin{aligned}
& u(F) \geq 2 \cdot \sup \{u(L) \mid L / K \text { finite extension }\} \\
& \ell(F) \geq 2 \cdot \sup \{u(L) \mid L / K \text { finite nonreal extension }\} .
\end{aligned}
$$

Proof: Let $L / K$ be a finite extension. Let $L_{0}$ denote the separable closure of $K$ inside $L$. As $L / L_{0}$ is purely inseparable, the natural map $W L_{0} \longrightarrow W L$ is an isomorphism by [20, Chap. VII, (2.6)] and because $L^{\times}=L_{0}^{\times} L^{\times 2}$. In particular $u(L)=u\left(L_{0}\right)$. As $L_{0} / K$ is a finite separable extension, $L_{0}$ is the residue field of a $\mathbb{Z}$-valuation $v$ on $F$. Using that $\operatorname{nrk}_{2}(v)=1$ if $L$ is nonreal by (3.3), the estimates now follow from (5.2).

6.2. Remark. For any finite real extension $L / K$ we obtain that $\ell(K(X)) \geq \ell(L)$, but this does not seem to be as useful as the second estimate in (6.1).

The following general facts will be needed in the comparison of the invariants $\alpha$ and $\beta$ and the stability index for a rational function field in (7.2) below.

6.3. Lemma. Any form that is Witt equivalent to a difference of two totally positive forms is isometric to a difference of two totally positive forms.

Proof: If $\varphi_{1}$ and $\varphi_{2}$ are totally positive forms over $K$ and $\varphi_{1} \perp-\varphi_{2}$ is isotropic, then there exists an element $a \in D_{K}\left(\varphi_{1}\right) \cap D_{K}\left(\varphi_{2}\right)$, and writing $\varphi_{i}=\psi_{i} \perp\langle a\rangle$ for $i=1,2$, also $\psi_{1}$ and $\psi_{2}$ are totally positive and $\left[\varphi_{1} \perp-\varphi_{2}\right]=\left[\psi_{1} \perp-\psi_{2}\right]$. This reduction argument readily yields the result.

6.4. Lemma. Assume that $I K \cdot\left(I_{t} K\right)^{n}=0$. Then $\left(I_{t} K\right)^{n}$ is generated as a group by the differences of totally positive $n$-fold Pfister forms over $K$. Moreover, any form in $\left(I_{t} K\right)^{n}$ is isometric to a difference of two totally positive forms. 
Proof: By $[4,(4.2)]$, we have in general that $\left(I_{t} K\right)^{n}$ is generated as a group by the elements $\left[a\left(\pi_{1} \perp-\pi_{2}\right)\right]$ with $a \in K^{\times}$and totally positive $n$-fold Pfister forms $\pi_{1}$ and $\pi_{2}$ over $K$. Assuming that $I K \cdot\left(I_{t} K\right)^{n}=0$, we further have that $\left[a\left(\pi_{1} \perp-\pi_{2}\right)\right]=\left[\pi_{1} \perp-\pi_{2}\right]$. This shows the first part, and the second part then follows with (6.3).

6.5. Lemma. Assume that $I_{t}^{2} K=\left(I_{t} K\right)^{2}$ and $I_{t}^{3} K=0$. Then any torsion binary form over $K$ represents an element of $\sum K^{2}$.

Proof: $\quad$ Since $I_{t}^{3} K=0$, we have in particular $I K \cdot\left(I_{t} K\right)^{2}=0$. Hence, (6.4) implies that for any $a \in K^{\times}$and $r \in \sum K^{2}$ we have $\langle\langle a, r\rangle\rangle=\langle\langle s, t\rangle\rangle$ for some $s, t \in \sum K^{2}$, and thus $a\langle 1,-r\rangle$ represents an element of $D_{K}\langle s, t\rangle \subseteq \sum K^{2}$.

6.6. Lemma. Assume that there exists $a \in K^{\times} \backslash \pm \sum K^{2}$ such that $a \in \pm \sum L^{2}$ for any proper finite extension $L / K$. Then $K$ is hereditarily pythagorean with four square classes, any finite extension of $K$ is a 2-extension, and $I_{t}^{3} K(X)=0$.

Proof: It is immediate from the assumption that $K$ is real and has no proper finite extension of odd degree. Thus every finite extension of $K$ is a 2-extension. For $b \in K^{\times} \backslash\left(-\sum K^{2} \cup a \sum K^{2} \cup-a \sum K^{2}\right)$, there exist orderings $P$ and $Q$ on $K$ with $a, b \in P$ and $-a, b \in Q$, and since both extend to $K(\sqrt{b})$ we have $a \notin \pm \sum K(\sqrt{b})^{2}$; in view of the assumption, this is only possible if $K(\sqrt{b})=K$. This argument shows that $\sum K^{2}=K^{\times 2}$ and $K^{\times}= \pm K^{\times 2} \cup \pm a K^{\times 2}$.

By (2.3) we conclude that any finite real extension of $K$ is pythagorean with four square classes and any finite nonreal extension of $K$ has two square classes. In particular $K$ is hereditarily pythagorean. Since $I_{t}^{2} L=0$ for any field $L$ that is either pythagorean or nonreal with two square classes, Milnor's Exact Sequence [20, Chap. IX, Sect. 3] yields that $I_{t}^{3} K(X)=0$.

6.7. Theorem. Let $K$ be a real field and $a \in K^{\times} \backslash \pm \sum K^{2}$. Then

$$
\left\langle\left\langle a, 1+X^{2}\right\rangle\right\rangle \in I_{t}^{2} K(X) \backslash\left(I_{t} K(X)\right)^{2} .
$$

Proof: Obivously, $\left\langle\left\langle a, 1+X^{2}\right\rangle\right\rangle$ is a torsion 2-fold Pfister form and thus lies in $I_{t}^{2} K(X)$. To show that $\left\langle\left\langle a, 1+X^{2}\right\rangle\right\rangle$ does not belong to $\left(I_{t} K(X)\right)^{2}$, we may enlarge $K$ while maintaining that $a \notin \pm \sum K^{2}$. Applying Zorn's Lemma, we may therefore reduce to the situation where $a \notin \pm \sum K^{2}$, whereas $a \in \pm \sum L^{2}$ for any proper finite extension $L / K$. Then (6.6) implies that $I_{t}^{3} F=0$ for $F=K(X)$ and that $K$ is hereditarily pythagorean, in particular $\sum F^{2}=D_{F}\langle 1,1\rangle$, by $[7$, Chap. III, Theorem 4] or [20, p. 397].

Suppose now on the contrary that $\left\langle\left\langle a, 1+X^{2}\right\rangle\right\rangle \in\left(I_{t} F\right)^{2}$. Then (6.4) applied with $n=2$ yields that $\left\langle\left\langle a, 1+X^{2}\right\rangle\right\rangle$ is a difference of two totally positive forms over $F$. It follows that $\left\langle\left\langle a, 1+X^{2}\right\rangle\right\rangle=\langle\langle s, t\rangle\rangle$ for some $s, t \in \sum F^{2}$. Then $D_{F}\left\langle a,-a\left(1+X^{2}\right)\right\rangle \cap D_{F}\langle s, t\rangle \neq \emptyset$, whence $a \in D_{F}\left\langle 1,-\left(1+X^{2}\right)\right\rangle \cdot D_{F}\langle s, t\rangle$. 
Since $D_{F}\langle s, t\rangle \subseteq \sum F^{2}=D_{F}\langle 1,1\rangle$, we conclude that $a$ is a sum of 2 squares in $F\left(\sqrt{-\left(1+X^{2}\right)}\right)$. In other words, the 2-fold Pfister form $\langle\langle-1, a\rangle\rangle$ becomes hyperbolic over $F\left(\sqrt{-\left(1+X^{2}\right)}\right)$. Note that $F\left(\sqrt{-\left(1+X^{2}\right)}\right)$ is $K$-isomorphic to the function field of the projective quadric given by the form $\langle 1,1,1\rangle$ over $K$. Using [20, Chap. X, (4.9)] it follows that the form $\langle\langle-1, a\rangle\rangle$ over $K$ is either hyperbolic or contains $\langle 1,1,1\rangle$ as a subform. This implies that $a \in \pm \sum K^{2}$, contradicting the hypothesis.

The following theorem extends and generalizes the well-known characterization of hereditarily euclidean fields via the rational function field.

6.8. Theorem. Assume that $K$ is real and let $F=K(X)$. For $n \geq 1$ the following are equivalent:

(i) $I^{n+1} F(\sqrt{-1})=0$;

(ii) $I_{t}^{n+1} F=0$;

(iii) $\left(I_{t} F\right)^{n+1}=0$;

(iv) $I_{t}^{n+1} F=\left(I_{t} F\right)^{n+1}$;

(v) $I_{t}^{n} L=0$ and $s t(L)<n$ for every finite real field extension $L / K$;

(vi) $I^{n} M=0$ for every finite field extension $M / K(\sqrt{-1})$.

For $n=1$ any of these conditions holds if and only if $K$ is hereditarily euclidean.

Proof: Regardless of the specific nature of the field $F$, we have $(i) \Rightarrow(i i)$ by (1.4), and both $(i i) \Rightarrow(i i i)$ and $(i i) \Rightarrow(i v)$ are obvious. Milnor's Exact Sequence [20, Chap. IX, (3.1)] yields $(i) \Leftrightarrow(v i)$.

For any finite nonreal extension $M / K$, as $M$ is the residue field of a $\mathbb{Z}$-valuation on $F=K(X)$, we have $\beta(M) \leq \beta(F)-1$ by (5.6). This shows $($ iii $) \Rightarrow(v i)$.

Consider now a finite extension $M / K(\sqrt{-1})$. Let $N / K$ be the normal closure of $M / K$ and let $L / K$ be a maximal real extension contained in $N / K$. Then $L(\sqrt{-1}) \subseteq N$. Assuming that $I_{t}^{n} L=0$ and $s t(L)<n$, we obtain that $I^{n} L(\sqrt{-1})=0$ by (1.4). Since $N / L(\sqrt{-1})$ is a 2-extension and $N / M$ is normal, by $\left[13,(4.5)\right.$ and (4.12)] this implies that $I^{n} N=0$ and $I^{n} M=0$. This shows $(v) \Rightarrow(v i)$.

It remains to show $(i v) \Rightarrow(v)$. Assume that $I_{t}^{n+1} F=\left(I_{t} F\right)^{n+1}$. For any finite real extension $L / K$, as $L$ is the residue field of a $\mathbb{Z}$-valuation on $F$, it follows by (5.8) applied with $m=n+1$ that $\alpha(L)<n$, hence $I_{t}^{n} L=0$.

Assume first that $n=1$. Then $K$ is hereditarily pythagorean. As $K$ is uniquely ordered by (6.7) it follows that $K$ is hereditarily euclidean by (2.6). Hence, $L$ is uniquely ordered and thus $s t(L)=0$.

Let now $n>1$. Consider a real valuation $v$ on $K$. Let $\widetilde{v}$ denote the extension of $v$ to a valuation on $F=K(X)$ determined by the equation

$$
\widetilde{v}\left(a_{0}+a_{1} X+\cdots+a_{r} X^{r}\right)=\max \left\{v\left(a_{0}\right), \ldots, v\left(a_{r}\right)\right\}
$$

for $r \in \mathbb{N}$ and $a_{0}, \ldots, a_{r} \in K$. Note that $\kappa_{\widetilde{v}}=\kappa_{v}(X)$ and $\Gamma_{\widetilde{v}}=\Gamma_{v}$, in particular $\operatorname{rk}_{2}(\widetilde{v})=\operatorname{rk}_{2}(v)$. Hence $\partial_{\widetilde{v}}\left(I_{t}^{n+1} F\right)=\partial_{\widetilde{v}}\left(I_{t} F\right)^{n+1}=\left(I_{t} \kappa_{v}(X)\right)^{n+1} \subseteq I_{t}^{2} \kappa_{v}(X)$ in 
view of the assumption and (5.4). It follows that $\operatorname{rk}_{2}(v) \leq n-1$, as otherwise there would exist a $\widetilde{v}$-balanced $n$-fold Pfister form $\pi$ over $F$ and then

$$
\left[\left\langle 1,-\left(1+X^{2}\right)\right\rangle\right]=\partial_{\widehat{v}}\left(\pi \otimes\left\langle 1,-\left(1+X^{2}\right)\right\rangle\right) \in I_{t}^{2} \kappa_{v}(X),
$$

which is impossible. Moreover, if $\operatorname{rk}_{2}(v)=n-1$, then

$$
I_{t}^{2} \kappa_{v}(X)=\partial_{\widetilde{v}}\left(I_{t}^{n+1} F\right)=\partial_{\widetilde{v}}\left(I_{t} F\right)^{n+1}=\left(I_{t} \kappa_{v}(X)\right)^{n+1} \subseteq\left(I_{t} \kappa_{v}(X)\right)^{2}
$$

by (5.7) and (5.4), and the implication proven already for $n=1$ yields that $\kappa_{v}$ is hereditarily euclidean.

Let $L / K$ be a finite real extension. The residue field of a valuation on $L$ is a finite extension of the residue field of its restriction to $K$. Hence, by the previous, for any real valuation $w$ on $L$, one has $\operatorname{rk}_{2}(w) \leq n-1$ and, if $\operatorname{rk}_{2}(w)=n-1$, then the residue field $\kappa_{w}$ is euclidean. By (4.5) this shows that $\operatorname{st}(L)<n$.

6.9. Remark. In $[4,(4.5)]$ we asked whether any real field $F$ with $I_{t}^{2} F=\left(I_{t} F\right)^{2}$ has Property $\left(S_{1}\right)$ introduced in [14], namely that every torsion binary form over $F$ represents a totally positive element. In the case where $F=K(X)$, we see from (6.8) and $[4,(3.8)]$ that both are equivalent with $K$ being hereditarily euclidean.

\section{FUNCTION FIELDS AND POWER SERIES FIELDS}

The following statement describes the behavior of our field invariants under a power series extension.

7.1. Proposition. Let $K$ be a real field and $L=K((X))$. For $n \geq 1$, one has $I^{n} L=I^{n} K+\langle 1, X\rangle \cdot I^{n-1} K, I_{t}^{n} L=I_{t}^{n} K+\langle 1, X\rangle \cdot I_{t}^{n-1} K$, and $\left(I_{t} L\right)^{n}=\left(I_{t} K\right)^{n}$. Furthermore, st $(L)=s t(K)+1, \alpha(L)=\alpha(K)+1, \beta(L)=\beta(K), \ell(L)=\ell(K)$, $u(L)=2 u(K)$, and $p(L)=p(K)$.

Proof: This follows from Springer's Theorem on quadratic forms over a complete discrete valued field [20, Chap. VI, Sect. 1]. The formula for the stability index is obtained from (4.1), using the stated equalities of ideals in $W L$.

Let $R$ in the sequel denote a real closed field.

7.2. Theorem. Let $F / R$ be a finitely generated extension and $n=\operatorname{td}(F / R)$.

(a) We have $\alpha(F)=\beta(F)=n$.

(b) If $F$ is real, then st $(F)=n$.

(c) If $n=1$, then $u(F)=\ell(F)=2$.

(d) If $n \geq 2$, then $2^{n} \leq \ell(F) \leq 2^{n}\left(2^{n-1}-1\right)+2$ and $2^{n} \leq u(F) \leq 2^{n+2}-2 n-6$.

Proof: We may assume that $n>0$. We fix a valuation on $F$ with value group $\mathbb{Z}$ and whose residue field a finite extension of $R(\sqrt{-1})\left(t_{1}, \ldots, t_{n-1}\right)$. Combining it with a valuation on the residue field with value group $\mathbb{Z}^{n-1}$, we obtain a valuation $v$ on $F$ with $\operatorname{nrk}_{2}(v)=n$. Therefore $u(F) \geq 2^{n}$ and $\ell(F) \geq 2^{n}$ by (5.2), and further $n \leq \beta(F) \leq \alpha(F) \leq \alpha(F(\sqrt{-1})$ ) by (5.6) and (1.4). If $F$ is real, then 
by $[9,(4.5)] F$ further carries a valuation $w$ with residue field $R$ and value group $\mathbb{Z}^{n}$, and it follows that $n=\operatorname{rk}_{2}(w) \leq \operatorname{st}(F) \leq \alpha(F(\sqrt{-1}))$ by (1.4).

As $R(\sqrt{-1})$ is algebraically closed, it follows using [25, Chap. 5, (1.4)] that $F(\sqrt{-1})$ is a $\mathcal{C}_{n}$-field in the terminology of Tsen-Lang Theory, in particular $u(F(\sqrt{-1})) \leq 2^{n}$. This implies that $\alpha(F(\sqrt{-1})) \leq n$, which finishes proving (a) and (b). In particular, if $n=1$, then $\alpha(F)=\beta(F)=1$, which is equivalent to saying that $u(F)=\ell(F)=2$, showing $(c)$. Finally, by [5, (6.1)] and [3], the upper bounds in part $(d)$ follow from the fact that $u(F(\sqrt{-1})) \leq 2^{n}$.

7.3. Theorem. Let $m, n \in \mathbb{N}$ with $m \leq n$ and

$$
F=R\left(\left(t_{1}\right)\right) \ldots\left(\left(t_{m-1}\right)\right)\left(t_{m}\right)\left(\left(t_{m+1}\right)\right) \ldots\left(\left(t_{n}\right)\right) .
$$

Then $p(F)=2, \beta(F)=m, \ell(F)=2^{m}$, st $(F)=\alpha(F)=\alpha(F(\sqrt{-1}))=n$, and $u(F)=u(F(\sqrt{-1}))=2^{n}$.

Proof: Using (7.1) we immediately reduce to the case where $m=n$ and thus $F=R\left(\left(t_{1}\right)\right) \ldots\left(\left(t_{n-1}\right)\right)\left(t_{n}\right)$. Then $F$ is a rational function field in one variable over the field $K=R\left(\left(t_{1}\right)\right) \ldots\left(\left(t_{n-1}\right)\right)$, which is hereditarily pythagorean. Hence, $p(F)=2$ by [7, Chap. III, Theorem 4] or [20, p. 397]. By (1.5) we have that $\operatorname{st}(F) \leq \alpha(F)=\beta(F)=\alpha(F(\sqrt{-1}))$.

Since $C=R(\sqrt{-1})$ is algebraically closed, it follows by [25, Chap. 5, (1.4) and $(2.2)]$ that $F(\sqrt{-1})=C\left(\left(t_{1}\right)\right) \ldots\left(\left(t_{n-1}\right)\right)\left(t_{n}\right)$ is a $\mathcal{C}_{n}$-field in the terminology of Tsen-Lang Theory. In particular, $u(F(\sqrt{-1})) \leq 2^{n}$ and thus $\alpha(F(\sqrt{-1})) \leq n$. As $p(F)=2$, using $[2,(3.5)]$ and $[5,(6.3)]$ we obtain that $u(F) \leq u(F(\sqrt{-1})) \leq 2^{n}$ and $\ell(F) \leq u(F(\sqrt{-1})) \leq 2^{n}$.

Let $v$ be the $K$-valuation on $F=K\left(t_{n}\right)$ associated to the polynomial $t_{n}^{2}+1$. Since $\kappa_{v}=C\left(\left(t_{1}\right)\right) \ldots\left(\left(t_{n-1}\right)\right)$ we have $u\left(\kappa_{v}\right)=\ell\left(\kappa_{v}\right)=2^{n-1}$, hence $u(F) \geq 2^{n}$ and $\ell(F) \geq 2^{n}$ by $(5.2)$. Hence, $\ell(F)=u(F)=u(F(\sqrt{-1}))=2^{n}$. Furthermore, as $F$ carries a real valuation with value group $\mathbb{Z}^{n}$, we have $s t(F) \geq n$ by (4.2). Therefore $\operatorname{st}(F)=\alpha(F)=\beta(F)=\alpha(F(\sqrt{-1}))=n$.

Acknowledgments. This work was supported by the Deutsche Forschungsgemeinschaft (project Quadratic Forms and Invariants, BE 2614/3-1) and by the Zukunftskolleg, Universität Konstanz.

\section{REFERENCES}

[1] J.K. Arason and R. Elman. Powers of the fundamental ideal in the Witt ring. J. Algebra 239 (2001): 150-160.

[2] K.J. Becher. On fields of $u$-invariant 4. Arch. Math. 86 (2006): 31-35.

[3] K.J. Becher. On the $u$-invariant of a real function field. Math. Ann. 346 (2010): 245-249.

[4] K.J. Becher, D.B. Leep. Pythagoras numbers and quadratic field extensions. Proceedings of the International Conference on the Algebraic and Arithmetic Theory of Quadratic Forms 2007, Lago Llanquihue (Chile). Contemp. Math. 493 (2009): 21-28.

[5] K.J. Becher, D.B. Leep. The length and other invariants of a real field. Math. Z., to appear. 
[6] K.J. Becher, D.B. Leep. The Elman-Lam-Krüskemper Theorem. ISRN Algebra, to appear.

[7] E. Becker. Hereditarily-pythagorean fields and orderings of higher level. Monografias de Matemática 29, Instituto de matematica pura e aplicada, Rio de Janeiro, 1978.

[8] E. Becker, E. Köpping. Reduzierte quadratische Formen und Semiordnungen reeller Körper. Abh. Math. Sem. Univ. Hamburg 46 (1977): 143-177.

[9] L. Bröcker. Zur Theorie der quadratischen Formen über formal reellen Körpern. Math. Ann. 210 (1974): 233-256.

[10] L. Bröcker. Characterization of Fans and Hereditarily Pythagorean Fields. Math. Z. 151 (1976): 149-163.

[11] R. Elman, N. Karpenko, A. Merkurjev. The algebraic and geometric theory of quadratic forms. American Mathematical Society Colloquium Publications, 56, Amer. Math. Soc., Providence, RI, 2008.

[12] R. Elman and T. Y. Lam. Quadratic forms and the u-Invariant, I. Math. Z. 131 (1973): $283-304$.

[13] R. Elman and T. Y. Lam. Quadratic forms under algebraic extensions. Math. Ann. 219 (1976): 21-42.

[14] R. Elman and A. Prestel. Reduced stability of the Witt ring of a field and its Pythagorean closure. Amer. J. Math. 106 (1984): 1237-1260.

[15] A.J. Engler, A. Prestel. Valued fields. Springer Monographs. Springer-Verlag, Berlin, 2005.

[16] B. Jacob. On the structure of Pythagorean Fields. J. Algebra, 68 (1981): 247-267.

[17] M. Knebusch. Specialization of quadratic and symmetric bilinear forms, and a norm theorem. Acta Arith. 24 (1973): 279-299.

[18] M. Krüskemper. On annihilators in graded Witt rings and in Milnor's $K$-theory. $W . B$. Jacob (ed.) et al., Recent advances in real algebraic geometry and quadratic forms. Proceedings of the RAGSQUAD year, Berkeley, CA, USA, 1990-1991. Providence, RI: American Mathematical Society. Contemp. Math. 155 (1994): 307-320.

[19] T.Y. Lam. Orderings, valuations and quadratic forms. CBMS Regional Conference Series in Math., Vol. 52. Amer. Math. Soc., Providence, RI, 1983.

[20] T.Y. Lam. Introduction to quadratic forms over fields. Graduate Studies in Mathematics, 67, Amer. Math. Soc., Providence, RI, 2005.

[21] M. Marshall. Some local global principles for formally real fields. Can. J. Math. 29 (1977): 606-614.

[22] M. Marshall. The Witt ring of a space of orderings. Trans. Amer. Math. Soc. 258 (1980): $505-521$.

[23] M. Marshall. Spaces of orderings and abstract real spectra. Lecture Notes in Mathematics, 1636. Springer-Verlag, Berlin, 1996.

[24] D. Orlov, A. Vishik, and V. Voevodsky. An exact sequence for $K_{*}^{M} / 2$ with applications to quadratic forms. (Ann. of Math. 165 (2007): 1-13.

[25] A. Pfister. Quadratic Forms with Applications to Algebraic Geometry and Topology. LMS Lecture Notes Series, 217. Cambridge University Press. Cambridge, 1995.

[26] A. Prestel. Lectures on Formally Real Fields. Lecture Notes in Mathematics, 1093, Springer-Verlag, Berlin, 1984.

Universität Konstanz, Zukunftskolleg / FB Mathematik und Statistik, 78457 Konstanz, Germany.

E-mail address: becher@maths.ucd.ie

Department of Mathematics, University of Kentucky, Lexington, KY 405060027, USA.

E-mail address: leep@email.uky.edu 